\title{
POLAR DECOMPOSITIONS OF BOUNDED LINEAR FUNCTIONALS ON OPERATOR SUBALGEBRAS
}

\author{
MASAHARU KUSUDA
}

(Communicated by Palle E. T. Jorgensen)

\begin{abstract}
Let $M$ be a von Neumann algebra and let $\varphi$ be a normal linear functional on a strongly closed $C^{*}$-subalgebra $N$ of $M$. Denote by $\mathscr{F}$ the set of normal linear functionals $\psi$ on $M$ extending $\varphi$ with $\|\psi\|=\|\varphi\|$. It is shown that there exists a partial isometry $v$ in $N$ such that

$$
\varphi=|\varphi|(v \cdot), \quad|\varphi|=\varphi\left(v^{*} \cdot\right), \quad\|\varphi\|=\||\varphi|\|
$$

and

$$
\psi=|\psi|(v \cdot), \quad|\psi|=\psi\left(v^{*} \cdot\right), \quad\|\psi\|=\||\psi|\|
$$

for all $\psi$ in $\mathscr{F}_{\varphi}$, where $|\varphi|$ and $|\psi|$ denote the absolute values of $\varphi$ and $\psi$ respectively. Let $A$ be a $C^{*}$-algebra and let $B$ be a $C^{*}$-subalgebra of $A$. As a consequence of this result, we obtain that every state on $B$ has a unique state extension to $A$ if and only if every bounded linear functional on $B$ has a unique norm-preserving extension to a bounded linear functional on $A$.
\end{abstract}

\section{INTRODUCTION}

Let $A$ be a $C^{*}$-algebra and let $B$ be a $C^{*}$-subalgebra of $A$. As is well known, every positive linear functional on $B$ has a norm-preserving extension to a positive linear functional on $A$. In general, however, such an extension is not unique. The uniqueness of such an extension would depend on the structure of $B$. Actually the author showed in [3, Theorem 2.2] that the following conditions are equivalent:

(1) $B$ is a hereditary $C^{*}$-subalgebra of $A$.

(2) Every positive linear functional on $B$ has a unique norm-preserving extension to a positive linear functional on $A$.

For the basic results of hereditary $C^{*}$-subalgebras, the reader is referred to [7]; for some recent results on hereditary $C^{*}$-subalgebras, to [2-6].

Recently Edwards and Rüttimann [1, Corollary 2.8] showed that the following conditions are equivalent:

(1) $B$ is a hereditary $C^{*}$-subalgebra of $A$.

(3) Every bounded linear functional on $B$ has a unique norm-preserving extension to a bounded linear functional on $A$.

Received by the editors October 29, 1991.

1991 Mathematics Subject Classification. Primary 46L10; Secondary 46L05, 46L30. 
As pointed out at the end of [1], conditions (2) and (3) are equivalent via condition (1).

In this paper, we shall give an elementary proof for the implication $(2) \Rightarrow(3)$, which is direct in the sense that we do not use condition (1). (The implication $(3) \Rightarrow(2)$ is clear since a positive linear functional on a $C^{*}$-subalgebra has a norm-preserving extension to a positive linear functional on the whole algebra.) Such a proof is deduced from the following theorem on polar decompositions of normal linear functionals on von Neumann algebras.

Theorem 2.1. Let $M$ be a von Neumann algebra and let $\varphi$ be a normal linear functional on a strongly closed $C^{*}$-subalgebra $N$ of $M$. Denote by $\mathscr{F}_{\varphi}$ the set of normal linear functionals $\psi$ on $M$ extending $\varphi$ with $\|\psi\|=\|\varphi\|$. Let $v$ be any partial isometry in $N$ satisfying that $\|\varphi\|=\varphi\left(v^{*}\right)$. Then we have

$$
\varphi=|\varphi|(v \cdot), \quad|\varphi|=\varphi\left(v^{*} \cdot\right), \quad\|\varphi\|=\||\varphi|\|
$$

and

$$
\psi=|\psi|(v \cdot), \quad|\psi|=\psi\left(v^{*} \cdot\right), \quad\|\psi\|=\||\psi|\|
$$

for all $\psi$ in $\mathscr{F}_{\varphi}$, where $|\varphi|$ and $|\psi|$ denote the absolute values of $\varphi$ and $\psi$ respectively.

This theorem suggests that research on norm-preserving extensions of bounded linear functionals on $C^{*}$-subalgebras should be reduced to research on norm-preserving positive extensions of positive linear functionals. In $\S 2$, we first prove this theorem, and the proof for $(2) \Rightarrow(3)$ is obtained as an easy consequence of this theorem. We emphasize that the key point in our obsevation is that for a normal linear functional $\varphi$ on a von Neumann algebra $M$, any partial isometry $v$ in $M$ with $\varphi(v)=\|\varphi\|$ always gives the polar decomposition of $\varphi$ (Lemma 2.3).

\section{Results}

Let $M$ be a von Neumann algebra and let $\varphi$ be a normal linear functional on $M$. By the polar decomposition of $\varphi$, we mean the following expression:

$$
\varphi=|\varphi|(v \cdot), \quad|\varphi|=\varphi\left(v^{*} \cdot\right)
$$

for some partial isometry $v$ in $M$ and a unique positive linear functional $|\varphi|$ on $M$ which satisfies

$$
\|\varphi\|=\||\varphi|\| \text { and }|\varphi(x)|^{2} \leq\|\varphi\||\varphi|\left(x^{*} x\right)
$$

for all $x$ in $M$ (cf. $[7,3.6 .7 ; 8,1.14 .4 ; 9$, III.4.2]). Note that condition (*) ensures the uniqueness of $|\varphi|$ (cf. [7, 3.6.7; 9, III.4.2]). More precisely, if a positive linear functional $\psi$ satisfies

$$
\|\psi\|=\||\varphi|\| \text { and }|\varphi(x)|^{2} \leqq\|\varphi\| \psi\left(x^{*} x\right),
$$

then $\psi=|\varphi|$. In addition, if $v v^{*}$ is exactly equal to the support projection $s(|\varphi|)$ of $|\varphi|$, i.e., the smallest of all projections $p$ in $M$ such that $|\varphi|(p \cdot)=$ $|\varphi|$, then $v$ is uniquely determined (cf. [8, 1.14.4; 9, III.4.6]). In general, however, there is some freedom for the choice of $v$. This is unfortunate in some cases, while in other cases it is fortunate. The starting point in this paper 
is Lemma 2.3. Although its proof is easily seen from [7, 3.6.8; 8, 1.14.4], we will give the proof below for the convenience of the reader, together with additional remarks.

Now we set

$$
M_{\varphi}=\{x \in M \mid\|x\| \leq 1, \varphi(x)=\|\varphi\|\},
$$

which is a nonempty and weakly compact face of the unit ball of $M$. Hence $M_{\varphi}$ contains a partial isometry in $M$, which is an extreme point of the unit ball of $M$. Note that the normal linear functional $\varphi(x \cdot)$ given by each $x$ in $\dot{M}_{\varphi}$ is positive. In fact, since

$$
\|\varphi(x \cdot)\| \geq \varphi(x 1)=\varphi(x)=\|\varphi\| \geq\|\varphi(x \cdot)\|,
$$

we obtain that $\|\varphi\|=\|\varphi(x \cdot)\|=\varphi(x 1)$, which implies that $\varphi(x \cdot)$ is positive (cf. $[7,3.14 ; 8,1.5 .2])$. In particular, $\varphi\left(v^{*} \cdot\right)$ is positive and $\|\varphi\|=\left\|\varphi\left(v^{*} \cdot\right)\right\|$ for all partial isometries $v^{*}$ in $M_{\varphi}$.

Lemma 2.2. Let $M$ be a von Neumann algebra and let $\varphi$ be a normal linear functional on $M$. Then

$$
\left\|\varphi\left(v^{*} v \cdot\right)\right\|=\|\varphi\|
$$

for every partial isometry $v^{*}$ in $M_{\varphi}$.

Proof. Since

$$
\|\varphi\| \geq\left\|\varphi\left(v^{*} v \cdot\right)\right\| \geq \varphi\left(v^{*} v v^{*}\right)=\varphi\left(v^{*}\right)=\|\varphi\|,
$$

we obtain the desired result. Q.E.D.

It is known that $\|\varphi(p \cdot)\|=\|\varphi\|$ for a projection $p$ in $M$ if and only if $\varphi(p \cdot)=\varphi$ (e.g., [9, III.4.1]). We thus see that $\varphi\left(v^{*} v \cdot\right)=\varphi$ for every partial isometry $v^{*}$ in $M_{\varphi}$. The next lemma asserts that $\varphi(v \cdot)=\varphi(w \cdot)$ for arbitrary isometries $v$ and $w$ in $M_{\varphi}$.

Lemma 2.3. Let $M$ be a von Neumann algebra and let $\varphi$ be a normal linear functional on $M$. Then we have

$$
\varphi=|\varphi|(v \cdot), \quad|\varphi|=\varphi\left(v^{*} \cdot\right), \quad\|\varphi\|=\||\varphi|\|
$$

for all partial isometries $v^{*}$ in $M_{\varphi}$.

Proof. In order to show that $\varphi\left(v^{*} \cdot\right)$ coincides with $|\varphi|$, we have only to verify that $\varphi\left(v^{*} \cdot\right)$ satisfies condition $(*)$. The Cauchy-Schwartz inequality shows that

$$
|\varphi(x)|^{2}=\left|\varphi\left(v^{*}(v x)\right)\right|^{2} \leq\left\|\varphi\left(v^{*} \cdot\right)\right\| \varphi\left(v^{*}\left(x^{*} v^{*} v x\right)\right) \leq\|\varphi\| \varphi\left(v^{*}\left(x^{*} x\right)\right)
$$

for all $x$ in $M$, which implies that $|\varphi|=\varphi\left(v^{*} \cdot\right)$, and we see that $\varphi(x)=$ $\varphi\left(v^{*} v x\right)=|\varphi|(v x)$. We thus complete the proof. Q.E.D.

Now we return to Theorem 2.1 and prove it.

Proof of Theorem 2.1. Take any partial isometry $v^{*}$ from $N_{\varphi}$. Since $N_{\varphi} \subset$ $M_{\psi}$ for all $\psi$ in $\mathscr{F}_{\varphi}$, it follows from Lemma 2.3 that $v$ yields the desired result. Q.E.D.

Let $A$ be a $C^{*}$-algebra. We denote by $A^{* *}$ the enveloping von Neumann algebra of $A$, which is identified with the second dual of $A$. Then $B^{* *}$ of a $C^{*}$-subalgebra $B$ of $A$ is identified with the strong closure of $B$ in $A^{* *}$. Thus we have the following. 
Corollary 2.4. Let $A$ be a $C^{*}$-algebra and let $\varphi$ be a bounded linear functional on a $C^{*}$-subalgebra $B$ of $A$. Denote by $\mathscr{F}_{\varphi}$ the set of bounded linear functionals $\psi$ on $A$ extending $\varphi$ with $\|\psi\|=\|\varphi\|$. Let $v^{*}$ be any partial isometry in $B_{\varphi}^{* *}$. Then we have

$$
\varphi=|\varphi|(v \cdot), \quad|\varphi|=\varphi\left(v^{*} \cdot\right), \quad\|\varphi\|=\||\varphi|\|
$$

and

for all $\psi$ in $\mathscr{F}_{\varphi}$.

$$
\psi=|\psi|(v \cdot), \quad|\psi|=\psi\left(v^{*} \cdot\right), \quad\|\psi\|=\||\psi|\|
$$

We are now in a position to prove the equivalence of (2) and (3) in the introduction.

Corollary 2.5 ([1]). Let $A$ be a $C^{*}$-algebra and let $B$ be a $C^{*}$-subalgebra of $A$. Then the following conditions are equivalent:

(1) Every positive linear functional on $B$ has a unique norm-preserving extension to a positive linear functional on $A$.

(2) Every bounded linear functional on $B$ has a unique norm-preserving extension to a bounded linear functional on $A$.

Proof. We have only to prove the implication (1) $\Rightarrow(2)$. Let $\varphi$ be a bounded linear functional on $B$ and let $\varphi_{1}$ and $\varphi_{2}$ be norm-preserving extensions of $\varphi$ to bounded linear functionals on $A$. It follows from Corollary 2.4 that there exists a partial isometry $v$ in $B^{* *}$ such that

$$
\varphi=|\varphi|(v \cdot), \quad|\varphi|=\varphi\left(v^{*} \cdot\right), \quad\|\varphi\|=\|\varphi\|
$$

and

$$
\varphi_{i}=\left|\varphi_{i}\right|(v \cdot), \quad\left|\varphi_{i}\right|=\varphi_{i}\left(v^{*} \cdot\right), \quad\left\|\varphi_{i}\right\|=\left\|\left|\varphi_{i}\right|\right\|
$$

for $i=1,2$. We then have

$$
\left|\varphi_{i}\right|(b)=\varphi_{i}\left(v^{*} b\right)=\varphi\left(v^{*} b\right)=|\varphi|(b)
$$

for all $b \in B$. Since we have

$$
\left\|\left|\varphi_{i}\right|\right\|=\left\|\varphi_{i}\right\|=\|\varphi\|=\||\varphi|\|,
$$

condition (1) concludes that $\left|\varphi_{1}\right|=\left|\varphi_{2}\right|$. This means that $\varphi_{1}=\left|\varphi_{1}\right|(v \cdot)=$ $\left|\varphi_{2}\right|(v \cdot)=\varphi_{2}$. We thus complete the proof. Q.E.D.

\section{ACKNOWLEDGMENTS}

The author would like to thank C. M. Edwards for useful discussions and for providing a copy of the preprint [1]. This research was done while the author was staying at The Mathematical Institute, University of Oxford. He also thanks C. J. K. Batty and The Mathematical Institute for their warm hospitality.

\section{REFERENCES}

1. C. M. Edwards and G. T. Rüttimann, A characterization of inner ideals in J $B^{*}$-triples, Proc. Amer. Math. Soc. (to appear).

2. M. Kusuda, Hereditary $C^{*}$-subalgebras of $C^{*}$-crossed products, Proc. Amer. Math. Soc. 102 (1988), 90-94. 
3. 201-209.

4. __ A characterization of ideals of $C^{*}$-algebras, Canad. Math. Bull. 33 (1990), 455-459.

5. Characterizations of certain classes of hereditary $C^{*}$-subalgebras, Proc. Amer. Math. Soc. (to appear).

6. __ Characterizations of hereditary $C^{*}$-subalgebras (in preparation).

7. G. K. Pedersen, $C^{*}$-algebras and their automorphism groups, Academic Press, London, 1979.

8. S. Sakai, $C^{*}$-algebras and $W^{*}$-algebras, Springer-Verlag, New York, Heidelberg, and Berlin, 1970.

9. M. Takesaki, Theory of operator algebras. I, Springer-Verlag, New York, Heidelberg, and Berlin, 1979.

Department of Applied Mathematics, Faculty of Engineering Science, Osaka UniverSITY, TOYONAKA, OSAKA 560, JAPAN

E-mail address: kusuda@sigmath.osaka-u.ac.jp 